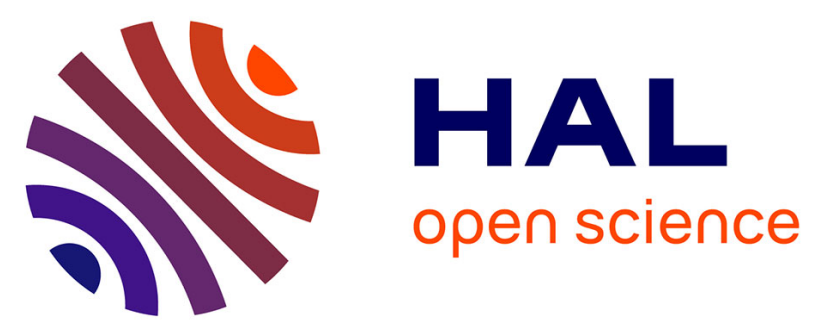

\title{
Towards the 3D characterisation of deformation micro-mechanisms within a compressed bundle of fibres
}

P. Latil, L. Orgéas, C. Geindreau, Pierre J.J. Dumont, S. Rolland Du Roscoat

\section{To cite this version:}

P. Latil, L. Orgéas, C. Geindreau, Pierre J.J. Dumont, S. Rolland Du Roscoat. Towards the 3D characterisation of deformation micro-mechanisms within a compressed bundle of fibres. Composites Science and Technology, 2011, 71 (4), pp.480. 10.1016/j.compscitech.2010.12.023 . hal-00723642

\section{HAL Id: hal-00723642 \\ https://hal.science/hal-00723642}

Submitted on 12 Aug 2012

HAL is a multi-disciplinary open access archive for the deposit and dissemination of scientific research documents, whether they are published or not. The documents may come from teaching and research institutions in France or abroad, or from public or private research centers.
L'archive ouverte pluridisciplinaire HAL, est destinée au dépôt et à la diffusion de documents scientifiques de niveau recherche, publiés ou non, émanant des établissements d'enseignement et de recherche français ou étrangers, des laboratoires publics ou privés. 


\section{Accepted Manuscript}

Towards the 3D in situ characterisation of deformation micro-mechanisms within a compressed bundle of fibres

P. Latil, L. Orgéas, C. Geindreau, P.J.J. Dumont, S. Rolland du Roscoat

PII:

S0266-3538(10)00491-4

DOI:

10.1016/j.compscitech.2010.12.023

Reference:

CSTE 4888

To appear in:

Composites Science and Technology

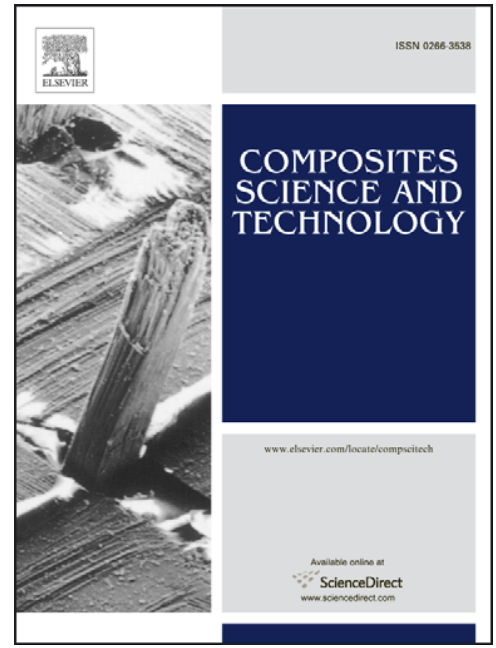

Received Date:

25 October 2010

Revised Date:

16 December 2010

Accepted Date:

18 December 2010

Please cite this article as: Latil, P., Orgéas, L., Geindreau, C., Dumont, P.J.J., du Roscoat, S.R., Towards the 3D in situ characterisation of deformation micro-mechanisms within a compressed bundle of fibres, Composites Science and Technology (2010), doi: 10.1016/j.compscitech.2010.12.023

This is a PDF file of an unedited manuscript that has been accepted for publication. As a service to our customers we are providing this early version of the manuscript. The manuscript will undergo copyediting, typesetting, and review of the resulting proof before it is published in its final form. Please note that during the production process errors may be discovered which could affect the content, and all legal disclaimers that apply to the journal pertain. 


\title{
Towards the 3D in situ characterisation of
}

\section{deformation micro-mechanisms within a}

\author{
compressed bundle of fibres
}

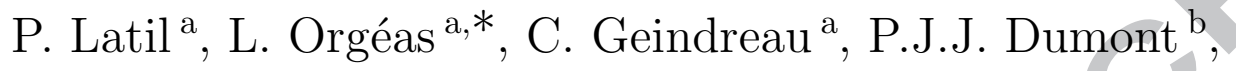 \\ S. Rolland du Roscoat ${ }^{\mathrm{a}, \mathrm{c}}$ \\ ${ }^{a}$ CNRS / Université de Grenoble, Laboratoire Sols-Solides-Structures-Risques \\ (3SR), BP 53, 38041 Grenoble cedex 9, France \\ ${ }^{\mathrm{b}}$ CNRS / Grenoble INP, Laboratoire de Génie des Procédés Papetiers (LGP2), \\ BP 65, 461 rue de la Papeterie, 38402 Saint-Martin d'Hères cedex 9, France \\ ${ }^{\mathrm{c}}$ European Synchrotron Radiation Facility (ESRF), ID 19 Topography and \\ Microtomography Group, 38043 Grenoble Cedex, France
}

\begin{abstract}
The present study provides original experimental data concerning the evolution of the microstructure of a bundle of fibres during its deformation. For that purpose, a model saturated fibre bundle was processed and was subjected to a compression loading by using a specially designed micro-compression rheometer which was mounted on a synchrotron X-ray microtomograph. Thus, 3D images of the evolving fibrous microstructure could be obtained. Results first show that the compression induced both the bundle consolidation and liquid phase migration. Secondly, (i) the position, the orientation, the displacement and the deformation of each fibre together with (ii) the position and the evolution of each fibre-fibre contact were followed during the compression. The in situ tracking of these microstructure descriptors allows the consolidation micro-mechanisms to be analysed and provides useful information for theoretical or numerical models used to predict fibre bundle deformation during processing of fibre bundle reinforced polymer composites.
\end{abstract}

Key words: A - Polymer Matrix Composites, A - Fibre, A - Fabrics/textiles, D X-ray microtomography, D - Rheology

\footnotetext{
* Corresponding author. Tel. : +33 476827073 ; Fax : +33 476827043

Email address: laurent.orgeas@grenoble-inp.fr (L. Orgéas).
} 


\section{Introduction}

In the past decades, the use of fibre-reinforced polymer composites in structural or functional applications has increased in various domains such as automotive or aerospace industries. Most of the fibrous reinforcements of these materials are made up of bundles containing few hundreds to few thousands continuous or discontinuous fibres. For this reason, these composite materials exhibit multiple scales: a macroscopic one, i.e. the part scale, a mesoscopic one, i.e. the fibre bundle scale, and a microscopic scale, i.e. the fibre scale. During their processing, e.g. sheet forming of long fibre reinforced thermoplatics, sheet forming of dry woven textiles for the Liquid Composite Moulding (LCM) processes, or compression molding of sheet moulding compounds, glass mat thermoplastics or hybrids, it is well known that the fibre bundles of these materials can be subjected to important displacements and deformations such as, for instance, orientation, consolidation, stretching, bending, twisting, shearing, bundle filamentation and breakage... [1-8]. These bundle mesoscale deformation mechanisms are induced both by the complex relative motions and the deformation of fibres at the microscopic level within the bundles. They drastically affect the rheological behaviour of the composites during their forming by modifying, for instance, the permeability and the anisotropy of the fibrous networks [5,9-14]. They also affect final physical and mechanical properties of produced parts. For those reasons, mesoscale deformation mechanisms of fibre bundles have been deeply studied experimentally by using various observation and characterisation techniques (see above references). All these crucial experimental data are very useful and partially contribute to the (in)validation of some of the assumptions stated in meso-macro or micro-macro modelling approaches dedicated to this type of fibrous media [6,15-18,20,21]. Among them, the X-ray microtomography technique which is now widely used in materials science $[22,23]$ is of particular interest since it can provide full $3 \mathrm{D}$ characterisa- 
tion of networks of fibres and their mesoscale deformation, even in composite materials with an optically opaque matrix. For instance, the orientation, the curvature, the compression and the breakage of discontinuous sized fibre bundles, occurring during the compression molding of an industrial SMC, were observed and quantified by using synchrotron X-ray microtomography with the phase contrast mode [7]. Similarly, by performing with dry woven fabrics in situ shear and biaxial tests inside a laboratory X-ray microtomograph, Badel et al [6] have underlined the very large shape and surface changes the cross sections of continuous fibre bundles of deformed textile reinforcements could be subjected to.

By contrast and surprisingly, deformation mechanisms at the fibre scale, which are however responsible for the mesoscale bundle deformation, still remain not very well understood. What is the relative motion of fibres contained inside a bundle when it is subjected to a mesoscale consolidating/dilating and/or shear loading? Meanwhile, do fibres stretch, bend or twist? What is their orientation evolution? How many fibre-fibre contacts are there in the bundle? Where are these contacts located? How do they evolve during the overall bundle deformation? What is their orientation? Can a fibre bundle be seen as a continuum at the mesoscale? Such type of crucial but still opened questions greatly hinder the development and the validation of the above meso-macro or micro-macro models. Discrete/finite element simulation performed at the fibre scale could be a relevant tool to answer them [17-19,24,25]. However, this type of approaches must be previously validated and compared with experimental data collected at the fibre scale. The objective of this contribution is thus to propose an experimental methodology for the quantitative analysis of deformation mechanisms occurring at the fibre scale when a bundle of fibres is subjected to a mechanical loading. In this preliminary work, the methodology is built and illustrated by compressing a model bundle of fibres (section 2), 
but other mechanical loading conditions and other fibre bundles could be used in future. The compression experiment is performed with a micro-rheometrer that is mounted in a synchrotron X-Ray microtomograph, which allows in situ observations of the bundle microstructures to be performed during the test. Therefrom, 3D relevant microstructure descriptors are quantified by using the 3D scanned images and image analysis subroutines (section 3). Thus, it is then possible to follow (section 4) and to discuss (section 5) the simultaneous evolutions of both the mesocopic mechanical response of the bundle and its associated microstructure descriptors.

\section{Material and experimental procedure}

In this preliminary study, the fibre bundle which was deformed was handmade. It consists of $N=69$ rather straight and rather aligned fibres of length $L=11 \mathrm{~mm}$ and diameter $D=150 \mu \mathrm{m}$. These fibres were extracted from a fluorocarbon (PVDF) continuous elastic fishing wire (tensile Young's modulus $E \approx 2 \mathrm{GPa}$ ). The bundle was saturated with olive oil which behaves as a low viscosity Newtonian fluid at room temperature (shear viscosity $\mu \approx 70 \mathrm{mPa}$ s) and which is solid below $5^{\circ} \mathrm{C}$. This property is interesting since it allows the freezing of the bundle fibrous microstructure: this facilitates the bundle handling before mechanical testing which was performed at room temperature, i.e. when the oil is a fluid.

The fibre bundle was subjected to a simple compression loading at a low strain rate, i.e. $\approx 510^{-3} \mathrm{~s}^{-1}$, with a compression micro-rheometer which was purposely designed. As shown in the scheme displayed in figure 1, the bundle was put between an upper fixed plate and a lower one which was actuated vertically (with a piezo-motor not shown here). Before starting the test, the

bundle was subjected to a very slight pre-compression, in order to 
ensure its upper and lower surfaces to be parallel with the rheometer plates. During this phase, no measurable force could be recorded. The end of this pre-compression was arbitrarily chosen as the initial state. During the test, the upper and lower faces of the bundle were subjected to a compression force $F_{2}$ along the $\mathbf{e}_{2}$-direction which was recorded with a load cell (maximum capacity $500 \mathrm{~N}$ ) together with the current height of the bundle $l_{2}$. The other surfaces of the bundle remained stress free. The compression loading was interrupted at five compression strains $\varepsilon_{22}=\ln \left(l_{2} / l_{20}\right), l_{20}$ being the initial height of the bundle. During these stops, slight decreases $(\approx 10 \%)$ of the compression force were observed for $\approx 30 \mathrm{~s}[3]$, corresponding to a rearrangement of the fibrous microstructure and the fluid. Afterwards, the force remained constant and 3D images of the fibrous microstructure could be obtained. For that purpose, the micro-rheometer was installed in the X-ray microtomograph of the ID19 beamline at the European Synchrotron Radiation Facilities (Grenoble, France). At each compression stop, a fast scan of the bundle was achieved (overall scanning time $\approx 1 \mathrm{~min}$ ) consisting in $1500 \mathrm{X}$-ray $2 \mathrm{D}$ projections obtained from the incremental rotation along $\mathbf{e}_{2}$ of the micro-rheometer with respect to the synchrotron X-ray source (overall rotation $=180^{\circ}$, beam energy $=25 \mathrm{keV}$ ). After proper reconstruction of the $2 \mathrm{D}$ projections, 3D maps of the X-ray absorption coefficient inside the bundle could be obtained (volumes of maximal size of $2048 \times 2048 \times 512$ voxels, voxel size $=7.5 \mu \mathrm{m})$. The maps were denoised by using suitable filtering operations which faded their noise/signal ratio induced by the very short exposure time. Then, these five 3D maps could be cut in order to get cross sections of the micro-rheometer and the bundle (see figure 1). They can also be used to characterise the bundle microstructure (see below). 


\section{Image analysis and data post-treatment}

To analyse quantitatively both the mesoscopic mechanical response and the microstructure of the bundle of fibres, the five scanned volumes were subjected to several image analysis operations achieved with the softwares ImageJ and Matlab. These operations are described below and summarised in figure 2.

\subsection{Volume of analysis - Mesoscale strains and stresses}

The evolving volume of fibres $V$, (i) from which the mesoscopic mechanical response was estimated and (ii) within which the microstructure parameters of the bundle were extracted and analysed, was chosen in order to follow the mesoscopic deformation of the fibre bundle. After a first observation of the 3D images acquired during the test, it was found that the bundle flow was alike a lubricated compression, where the flow along the $\mathbf{e}_{3}$-direction was negligible compared to that in the $\left(\mathbf{e}_{1}, \mathbf{e}_{2}\right)$ plane. From this observation, $V$ was chosen as a rectangular box (see figures 1 and 2(b)): its current height $l_{2}$ was given as the distance between the two plates of the micro-rheometer, its current width $l_{1}$ was set in order to contain all the fibres of the bundle and its length $l_{3}$ was set constant. As shown in figure 1(b), notice that due to the heterogeneity of fibres' lengths (induced by the manufacturing protocol), $l_{3}$ was such that it slightly and arbitrarily truncates (about $1 \mathrm{~mm}$ ) the ends of some of the longest fibres which are not involved in the mechanical response of the bundle. It was then possible to estimate the mean strain the bundle was subjected to. For instance, the mean Hencki strain tensor $\boldsymbol{\varepsilon}$ could be estimated:

$$
\varepsilon \approx \varepsilon_{11} \mathbf{e}_{1} \otimes \mathbf{e}_{1}+\varepsilon_{22} \mathbf{e}_{2} \otimes \mathbf{e}_{2} \quad \text { with } \quad \varepsilon_{11} \approx \ln \frac{l_{1}}{l_{10}} \quad \text { and } \quad \varepsilon_{22} \approx \ln \frac{l_{2}}{l_{20}}
$$


together with its associated volume change $\varepsilon_{v}$ and deviatoric strain $\varepsilon_{d}$ :

$$
\varepsilon_{v}=\varepsilon: \delta, \quad \varepsilon_{d}=\sqrt{\frac{2}{3}\left(\varepsilon-\frac{\varepsilon_{v}}{3} \boldsymbol{\delta}\right):\left(\varepsilon-\frac{\varepsilon_{v}}{3} \boldsymbol{\delta}\right)},
$$

where $\boldsymbol{\delta}$ is the identity tensor. Furthermore, as the bundle was deformed at a very low strain rate with a very low viscosity saturating fluid, its compression could reasonably be considered as a drained compression during which interstitial fluid pressure effects are negligible: the monitored compression force was principally related to the microscale deformation of the fibres and their contacts. From this remark and from the estimation of the current volume of the bundle $V$, it is possible to estimate the mean Cauchy stress tensor of the bundle $\boldsymbol{\Sigma}$ as

$$
\boldsymbol{\Sigma}=\Sigma_{22} \mathbf{e}_{2} \otimes \mathbf{e}_{2} \quad \text { with } \quad \Sigma_{22} \approx \frac{F_{2}}{l_{1} l_{3}} .
$$

\subsection{Detection / Description of fibres}

Within the volume $V$, a standard thresholding operation was achieved in order to extract the fibre phase from the grey scale images (figure 2(a)), as illustrated in the binarised 3D views shown in figure 2(b). Hence, a first relevant microstructure descriptor, i.e. the volume fraction of fibres $\Phi$, was simply estimated by the ratio [number of voxels of the fibrous phase] / [number of voxels contained in $V]$. To analyse possible microstructure heterogenities, the volume fraction of fibre $\Phi^{\prime}$ in the core of the bundle was also estimated similarly, by choosing a volume $V^{\prime}$ centred in $V$ and such that $l_{1}^{\prime}=l_{1} / 3, l_{2}^{\prime}=l_{2}$ and $l_{3}^{\prime}=l_{3}$.

In order to detect the centrelines of all the fibres contained in $V$ from the previous binarised images (see figure $2(\mathrm{c})$ ), a procedure which is very efficient for almost parallel fibres with convex cross sections was used. Firstly, as depicted in figure $3(\mathrm{~d})$, eleven planar slices parallel to the $\left(\mathbf{e}_{1}, \mathbf{e}_{2}\right)$ plane and separated by a distance of 110 voxels were extracted from the scanned volumes. Secondly, 
on each 2D slice, adjoining fibre cross sections were separated by a standard 2D watershed algorithm without major difficulties, as shown in figure 3(a-b). Thirdly, the position of the centre of mass of each fibre cross section was calculated automatically and labellised (see figure $3(\mathrm{~b}-\mathrm{c})$ ). Notice that due to the chosen resolution, the accuracy of their positions was about $\pm 7.5 \mu \mathrm{m}$. If this had negligible impact on the characterisation of fibre centrelines, this induced noticeable discrepancies on the detection of fibre-fibre contacts (see next subsection). Thirdly, the eleven detected centres of mass belonging to a given fibre $i$ were used to form its centreline (this operation was done manually), here discretised by a piecewise linear 3D parametric curve $\mathbf{x}^{i}\left(s^{i}\right)$, $s^{i}$ being the curvilinear abscissa of the considered fibre $i$ ( $c f$. figure $3(\mathrm{e})$ ). Therefrom, the fibrous medium was entirely reconstructed numerically (see figure $2(\mathrm{~d})$ ), by assuming that the fibre diameter $D$ was constant. It was then possible to estimate numerically the direction of the lowest inertia axis $\overline{\mathbf{p}}^{i}$ of each fibre $i$. For the studied bundle, where the fibres are rather straight, $\overline{\mathbf{p}}^{i}$ can be considered as a relevant measure of the mean orientation of the fibre $i$. Each centreline $i$ was also associated with a local Frenet basis $\left(\mathbf{t}^{i}, \mathbf{n}^{i}, \mathbf{b}^{i}\right)$. This constitutes a possible way to characterise the local geometry of the centreline $i$ (figure 3(f)). The tangent, normal and binormal unit vectors of this basis are respectively defined as (no summation on the indice $i$ ):

$$
\mathbf{t}^{i}=\frac{\mathrm{d} \mathbf{x}^{i}}{\mathrm{~d} s^{i}}, \quad \mathbf{n}^{i}=\frac{1}{\left\|\mathrm{~d} \mathbf{t}^{i} / \mathrm{d} s^{i}\right\|} \frac{\mathrm{d} \mathbf{t}^{i}}{\mathrm{~d} s^{i}}, \quad \mathbf{b}^{i}=\mathbf{t}^{i} \times \mathbf{n}^{i} .
$$

They are also linked by the Frenet-Serret formulae (no summation on $i$ ):

$$
\frac{\mathrm{d} \mathbf{t}^{i}}{\mathrm{~d} s^{i}}=\kappa^{i} \mathbf{n}^{i}, \quad \frac{\mathrm{d} \mathbf{n}^{i}}{\mathrm{~d} s^{i}}=-\kappa^{i} \mathbf{t}^{i}+\tau^{i} \mathbf{b}^{i}, \quad \frac{\mathrm{d} \mathbf{b}^{i}}{\mathrm{~d} s^{i}}=-\tau^{i} \mathbf{n}^{i},
$$

where $\kappa^{i}$ and $\tau^{i}$ are the local geometrical curvature and torsion of the fibre centreline $i$ at its curvilinear abscissa $s^{i}$. In this work, a finite difference scheme was used to estimate the local Frenet bases, the geometrical curvatures and torsions from the discretised representation of the fibre cen- 
trelines. Lastly, let us remark that the geometrical torsion is not equivalent to the mechanical twist of the fibres. It is the rate of change of the osculating plane $\left(\mathbf{t}_{i}, \mathbf{n}_{i}\right)$ of the fibre centreline $\mathbf{x}^{i}\left(s^{i}\right)$. If it is different of zero, the centreline is not a $2 \mathrm{D}$ curve.

\subsection{Detection / Description of fibre-fibre and fibre/plate contacts}

A fibre-fibre contact, e.g. like the one displayed in figure 2(e), was detected as soon as the local distance $d^{j}$ between two neighbouring fibre centrelines was equal to or below a given contact distance $h$. Therefrom, in order to characterise locally the contact, a local normal unit vector $\mathbf{q}^{j}$ corresponding to the local normal contacting plane and a local contact position $\mathbf{y}^{j}$ were associated with $d^{j}$. A similar procedure was achieved to detect the fibre-plate contacts, with a contact distance equal to $h / 2$. To account for the uncertainty on the determination of the positions of the fibre centrelines (see previous subsection), three contact distances $h$ were used to detect fibre-fibre contacts: the fibre diameter $D$ and $D \pm 7.5 \mu \mathrm{m}$. Each contact $j$ was then characterised by (i) its length $l^{j}$ along the centrelines of the considered contacting fibres (see figure 2(e)), (ii) its average contact distance $\bar{d}^{j}$, its average spatial position $\overline{\mathbf{y}}^{j}$ and (iii) its average orientation $\overline{\mathbf{q}}^{j}$ (see figure $2(\mathrm{e})$ ) (the indice "0" related to $l_{0}^{j}$ and $d_{0}^{j}$ in figure $2(\mathrm{e})$ and in the following correspond to the initial state).

\section{Results}

\subsection{Mesoscale mechanical behaviour}

The mesoscale mechanical response of the bundle is summarised in figure 4 .

This figure first shows that during its compression the fibre bundle is subjected 
to a pronounced consolidation. Indeed, its volumetric strain $\varepsilon_{v}$ is negative, its magnitude is as high as the deviatoric strain $\varepsilon_{d}$ and raises up to 0.45 . This is accompanied with a drastic increase of the mean volume fraction of fibres $\Phi$ from $\Phi_{0}=0.29$ to 0.45 and, consequently, with the migration of the fluid out of the bundle. Within the core of the bundle, i.e. in $V^{\prime}$, a similar trend is observed, but the volume fraction of fibres is higher: during the test, $\Phi^{\prime}$ goes from $\Phi_{0}^{\prime}=0.39$ (showing the initial heterogeneity of the bundle) to 0.58 . Finally, figure 4 also shows that the mean compression stress $\Sigma_{22}$ exhibits a significant hardening which is an increasing function of $\Phi$.

\subsection{In situ characterisation of fibres}

(i) Orientation - Figure 5(a-b) gives the fibre orientation in the initial and the final steps. Two representations of the fibre orientation are used in this figure. The first one is the richest and uses unit spheres. On the surfaces of the spheres, spots represent the extremities of the $N$ orientation vectors $\overline{\mathbf{p}}^{i}$, their origin being located at the centre of the sphere. The second one, more compact, is the second order fibre orientation tensors $\mathbf{A}^{f}$ [26], defined here in its discrete form as

$$
\mathbf{A}^{f}=\frac{1}{N} \sum_{i=1}^{N} \overline{\mathbf{p}}^{i} \otimes \overline{\mathbf{p}}^{i} .
$$

As evident from figure 5(a), both representations show that in the initial state, fibres are mostly aligned along the $\mathbf{e}_{3}$ direction, and that the disorientation is more pronounced in the plane $\left(\mathbf{e}_{1}, \mathbf{e}_{3}\right)$ than in the $\left(\mathbf{e}_{1}, \mathbf{e}_{2}\right)$ one. This figure also proves that these two trends are slightly accentuated during the compression.

(ii) Geometrical torsions and curvatures - The calculation of geometrical torsions $\tau^{i}$ proved that the average geometrical torsion $\bar{\tau}$ is negligible, i.e. below $10^{-18} \mathrm{~m}^{-1}$. This is not the case for the curvature. To illustrate this, the

evolutions of the mean local radii of curvature $\bar{R}^{i}=1 / \bar{\kappa}^{i}$ with the 
position $x_{3}$ along the bundle have been plotted in figure $6(\mathrm{a})\left(\bar{\kappa}^{i}\right.$ represents the average of the $\kappa^{i}$ estimated at $x_{3}$ for all the fibres in the bundle). This graph first shows that in the initial non-deformed state, fibres already exhibit finite curvature radii, with a mean value close to $50 \mathrm{~mm}$. This is expected because the fluorocarbon fishing wire, from which the fibres were extracted, was initially rolled onto a $25 \mathrm{~mm}$ radius reel and thus presented an initial curved shape at rest, which mainly contributes to the initial curvatures observed in figure 6 [27]. The graph also points out that whatever the compression state, the curvature radii $\bar{R}^{i}$ are more or less constant and with values close to the average curvature radius $\bar{R}$. Figure $6(\mathrm{~b})$ lastly proves that the local bending of fibres is drastically increased during the compression, since $\bar{R}$ is decreased from approximately $50 \mathrm{~mm}$ to $20 \mathrm{~mm}$ at the end of the test. This can also be observed qualitatively by looking at, for instance, the two contacting fibres shown in figure 2(e): these fibres are much more bent locally in the final compression state than in the initial one.

\subsection{In situ characterisation of contacts}

(i) Spatial distribution - As illustrated qualitatively in figure 5(c) and (d), fibre-fibre contacts are preferentially located in the core of the bundle, i.e. within $V^{\prime}$, where the fibre content is higher.

(ii) Number - The number of contacts in $V, \mathcal{N}$, substantially increases during the compression, from 61 to 239 (when $h=D$ ). To illustrate this, figure 7(a) shows the evolution of the average number of contacts per fibre or equivalently the average coordination number $\bar{z}=2 n^{c} / n^{f}$ as a function of $\Phi, n^{c}=\mathcal{N} / V$ and $n^{f}=4 \Phi / \pi D^{2} L$ being the numbers of contacts and fibres per unit volume, respectively: $\bar{z}$ undergoes a non linear increase from $\approx 2.2$ to $\approx 8.2$ as $\Phi$ goes from 0.29 to 0.45 . If the trend is preserved, noticeable shifts are observed by 
using $h=D \pm 7.5 \mu \mathrm{m}$, as emphasised by the error bars. Similar conclusions can be drawn by considering the average coordination number $\bar{z}^{\prime}$ in $V^{\prime}$ (figure $7(\mathrm{~b}))$. However, in accordance with the previous point (see figure $5(\mathrm{c}-\mathrm{d})$ ), this graph proves that $\bar{z}^{\prime}$ is higher than $\bar{z}$.

(iii) Orientation - The unit spheres displayed in figure 5(e-f), together with the second order contact orientation tensors $\mathbf{A}^{c}$ defined as

$$
\mathbf{A}^{c}=\frac{1}{\mathcal{N}} \sum_{j=1}^{\mathcal{N}} \overline{\mathbf{q}}^{j} \otimes \overline{\mathbf{q}}^{j}
$$

first prove that contacts are mostly orientated in the $\left(\mathbf{e}_{1}, \mathbf{e}_{2}\right)$ plane: this is mainly due to the weak misalignment of fibres. Furthermore, these two orientation representations underline the preferential orientation of contacts parallel to the compression direction $\mathbf{e}_{2}$, whatever the considered compression state: for instance, $A_{22}^{c}>A_{11}^{c}$.

(iv) Contact geometry - Figure 7(c) gives the evolution of the dimensionless average contact distance $\bar{d} / \bar{d}_{0}$ with $\Phi$ : this distance slightly decreases during the first steps of compression, the decrease is sharper for the last two steps and reaches $\approx 4 \%$ at the end of the test. By the same time, the average contact length $\bar{l} / \bar{l}_{0}$ noticeably diminishes during the compression, up to 0.67 (see figure $7(\mathrm{~d})$ ), even if this trend seems to be reversed at the last compression step. It is interesting to notice that the error bars induced by the choice of $h$ are smaller for these two descriptors than those obtained for $\bar{z}$ and $\bar{z}^{\prime}$.

\section{Discussion}

The mesoscopic mechanical response of the studied bundle, which is characterised by marked consolidation and hardening effects ( $c f$. figure 4 ), is a behaviour that is commonly observed during the compression of dry/saturated 
disordered/ordered fibrous media made up of contacting elastic fibres $[1,4,15]$. In particular, the experimental data plotted in figure 4(b) can be fitted by the following power-law type expression which is often proposed [15]:

$$
\Sigma_{22}=k E\left(\Phi^{m}-\Phi_{0}^{m}\right)
$$

The two constitutive parameters $k$ and $m$ were here set to 54.8 and 14.25 , respectively: the value of $m$ is consistent with those reported in the literature for similar fibrous media, i.e. $7 \leq m \leq 16$ [15]. Beyond these phenomenological considerations, it is important to notice that trends reported in figure 4 are directly connected to the actual microstructure of the bundle and its evolution, which is ruled by micro-mechanisms arising at the fibre seale. By using standard micro-meso theories dedicated to discrete media (granular or fibrous), it would be possible, in principle, to account for these microscale effects and predict their mesoscale behaviour. For instance, the mesoscale stress tensor $\Sigma$ of a fibrous medium containing $\mathcal{N}_{c}$ fibre-fibre contacts inside a representative volume $V_{f}$ can be expressed as $[15-17,21]$ :

$$
\Sigma=\frac{1}{V_{f}} \sum_{j=1}^{\mathcal{N}_{c}} \boldsymbol{\xi}^{j} \otimes \mathbf{f}^{j}
$$

where $\mathbf{f}^{j}$ is the contact force at the contact $j$, between a fibre $i$ of centre of mass $G^{i}$ and a fibre $k$ of centre of mass $G^{k}$, and where $\boldsymbol{\xi}^{j}=\mathbf{G}^{i} \mathbf{G}^{k}$. In regard to the current experimental work, this expression brings up the following comments:

(i) The role of the fibrous microstructure on the mesocopic behaviour is obviously underlined from (9). In particular, such an expression shows that stress levels depend on the relative positions of contacting fibres, i.e. $\boldsymbol{\xi}^{j}$, which in turn depends on the fibre geometry (aspect ratio, cross section, waviness), content and orientation. Similarly, (9) also emphasises the role of the number of contacts $\mathcal{N}_{c}$ in $V_{f}$, or equivalently the coordination numbers $Z$ in $V_{f}$. The method proposed in this study is a possible "experimental way" to get estimates of 
these descriptors (e.g. figures 5-7). Nonetheless, this has to be achieved cautiously. For example, it was shown that the bundle displayed heterogeneous fibre content along its width and that this heterogeneity was preserved during the consolidation: this partially explains why the mean coordination number in $V^{\prime}$, i.e. $\bar{z}^{\prime}$, is higher than the one in $V$, i.e. $\bar{z}(c f$. figure $7(\mathrm{a}-\mathrm{b}))$. Edge effects at the lateral free surfaces $x_{1}= \pm l_{1} / 2$ could also contribute to this observation.

The method also provides experimental evidences to (in)validate microstructure models of the literature. Among them, one of the simplest is the soft core tube model $[15,17,28,29]$. By assuming that (a) fibres are uniformly distributed in the space and (b) can be seen as straight cylinders that can overlap, the tube model provides, for instance, an estimate of the average coordination number $\bar{Z}$ in an volume $V_{f}$ containing a sufficient number of fibres $N_{f}$ :

$$
\bar{Z}=4 \phi\left(\frac{2}{\pi} r \frac{1}{N_{f}^{2}} \sum_{i=1}^{N_{f}} \sum_{k=1}^{N_{f}}\left\|\overline{\mathbf{p}}^{i} \times \overline{\mathbf{p}}^{k}\right\|+\frac{1}{N_{f}^{2}} \sum_{i=1}^{N_{f}} \sum_{k=1}^{N_{f}}\left|\overline{\mathbf{p}}^{i} \cdot \overline{\mathbf{p}}^{k}\right|+1\right)
$$

This expression emphasises the role of the fibre aspect ratio $r=L / D$, orientation $\overline{\mathbf{p}}^{i}$ and content $\Phi$ on $\bar{Z}$. It was used to estimate the average coordination numbers $\bar{z}$ and $\bar{z}^{\prime}$, by setting respectively in it $\phi$ to $\Phi$ and $\Phi^{\prime}, N_{f}$ to $N$ and $N^{\prime}$, and by using the measured value of the orientation vectors $\overline{\mathbf{p}}^{i}$ in $V$ and $V^{\prime}$, respectively. If the experimental trend is more or less reproduced, figure $7(a-b)$ shows that the analytical tube model (10) largely overestimates the experimental coordination numbers. This is mainly due to the tested bundle which does not fulfil the hypothesis (a) of the tube model. Indeed, within $V$ and $V^{\prime}$, fibres are not homogeneously distributed spatially, in particular along the $\mathbf{e}_{3}$-direction. This trend is enhanced in $V$ since noticeable variations of the fibre content are recorded (e.g. $\left.\Phi<\Phi^{\prime}\right)$. Besides, experimental estimations of $\bar{z}$ and $\bar{z}^{\prime}$ were achieved with a low number of fibres and by accounting for possible edge effects at fibre-plate contacts. This is further enhanced for $\bar{z}$, since additional edge effects might occur at the free surface of the bundle, i.e. at 
$x_{1}= \pm l_{1} / 2$ (see above). By forgetting the assumption (a) of the tube model, i.e. by reconstructing numerically a bundle with $N$ straight overlapping cylinders, the centres of mass and the orientations of which correspond to those measured in the tested bundle, figure $7(\mathrm{a}-\mathrm{b})$ shows that predictions of the tube model (without assumption (a)) are much closer to experimental values. However, due to the simplicity of assumption (b), i.e. soft core model plus no bending of fibres, the predicted coordination numbers remain overestimated.

(ii) The role of the deformation micro-mechanisms in (9) is also emphasised from the contact forces $\mathbf{f}^{j}$ which contribute to the transfer, from fibre to fibre, of the mesoscopic compression loading. Firstly, contact forces induce the bending of fibres between consecutive contacts when fibres are not properly aligned: this explains the trends emphasised in figures 2(e) and 6. Secondly, while increasing the mesoscopic loading, load transfers from fibre to fibre also lead to deform contact zones and increase, in case of elastic fibres, the magnitude contact forces $\mathbf{f}^{j}$. These deformation mechanisms, together with the increase of the coordination numbers $\bar{z}$ or $\bar{z}^{\prime}$ (figure $7(\mathrm{a}-\mathrm{b})$ ), contribute to the trends reported in figure 4 , i.e. the severe hardening of stress levels, the bundle consolidation and the drainage of the fluid. In the present study, it is interesting to notice that such mechanisms may be quite complex. For example, figure $7(\mathrm{c}-\mathrm{d})$ shows that the contact distance $\bar{d} / \bar{d}_{0}$ and, more surprisingly, the contact length $\bar{l} / \bar{l}_{0}$ were decreasing during the compression. The last decrease could be due to the slight misalignment of fibres observed during the compression (figure $5(\mathrm{a}-\mathrm{b}))$.

\section{Conclusion}

The preliminary results that have been obtained in this study are very encouraging. By combining a mechanical test on a fibrous medium, in situ 3D 
observations of its evolving microstructure and suitable image analysis subroutines, they prove that it was possible to characterise simultaneously meso but also micro deformation mechanisms in this fibrous medium. We have shown with some examples that obtained results could provide useful information to understand the mechanics of fibrous media, but also to validate theoretical frameworks dedicated to the modelling of their microstructure and deformation. Future work will enter more deeply in this process, (i) by studying various types of fibrous media, (ii) by subjecting them to various types of mesoscale mechanical loading, (iii) by estimating quantitatively the mesoscale stress tensor $(9)$.

Acknowledgements - This work was performed within the ANR research program "3D discrete Analysis of micromechanisms of deformation in highly concentrated fibre suspensions" (ANAFIB, ANR-09-JCJC-0030-01) and the ESRF Long Term Project "Heterogeneous Fibrous Materials". The authors also thank the french region Rhône-Alpes for its financial support.

\section{References}

[1] F. Robitaille, R. Gauvin, Compaction of textile reinforcements for composites manufacturing. i: Review of experimental results, Polym. Compos. 19 (1998) 198-216.

[2] K. Buet-Gautier, P. Boisse, Experimental analysis and modeling of biaxial mechanical behavior of woven composite reinforcements, Exp. Mech. 41 (2001) 260-269.

[3] S. Bickerton, M. Buntain, A. Somashekar, The viscoelastic compression behavior of liquid composite molding preforms, Compos. Part A 34 (2003) 431444.

[4] S. Comas-Cardona, P. Le Grognec, C. Binetruy, P. Krawczak, Unidirectional compression of fibre reinforcements. part 1: A non-linear elastic-plastic behaviour, Compos. Sci. Technol. 67 (2007) 507-514.

[5] P. Dumont, J.-P. Vassal, L. Orgéas, V. Michaud, D. Favier, J.A-E. Månson, Processing, characterization and rheology of transparent concentrated fibre 
bundle suspensions, Rheol. Acta 46 (2007) 639-651.

[6] P. Badel, E. Vidal-Salle, E. Maire, P. Boisse, Simulation and tomography analysis of textile composite reinforcement deformation at the mesoscopic scale, Compos. Sci. Technol. 68 (2008) 2433-2440.

[7] T.H. Le, P. Dumont, L. Orgéas, D. Favier, L. Salvo, E. Boller, X-ray phase contrast microtomography for the analysis of the fibrous microstructure of SMC composites, Compos. Part A 39 (2008) 91-103.

[8] S. Lomov, P. Boisse, E. Deluycker, F. Morestin, K. Vanclooster, D. Vandepitte, I. Verpoest, A. Willems, Full-field strain measurements in textile deformability studies, Compos. Part A 39 (2008) 1232-1234.

[9] C. Lai, W. Young, Model resin permeation of fibre reinforcements after shear deformation, Polym. Compos. 18 (1997) 6428.

[10] P. Smith, C. Rudd, A. Long, The effect of shear deformation on the processing and mechanical properties of aligned reinforcements, Compos. Sci. Technol. 57 (1997) 327-344.

[11] M. Buntain, S. Bickerton, Compression flow permeability measurement: a continuous technique, Compos. Part A 34 (2003) 445-457.

[12] S. Comas-Cardona, C. Binetruy, P. Krawczak, Unidirectional compression of fibre reinforcements. Part 2: A continuous permeability tensor measurement, Compos. Sci. Technol. 67 (2007) 638-645.

[13] F. Loix, P. Badel, L. Orgéas, C. Geindreau, P. Boisse, Woven fabric permeability: from textile deformation to fluid flow mesoscale simulations, Compos. Sci. Technol. 68 (2008) 1624-1630.

[14] F. Loix, L. Orgéas, C. Geindreau, P. Badel, P. Boisse, J.-F. Bloch, Flow of non-Newtonian liquid polymers through deformed composite reinforcements, Compos. Sci. Technol. 69 (2009) 612-9

[15] S. Toll, Packing mechanics of fiber reinforcements, Polym. Eng. Sci. 38 (1998) 1337-1350.

[16] S. Le Corre, D. Caillerie, L. Orgéas, D. Favier, Behavior of a net of fibers linked by viscous interactions: Theory and mechanical properties, J. Mech. Phys. Solids 52 (2004) 395-421.

[17] S. Le Corre, P. Dumont, L. Orgéas, D. Favier, Rheology of highly concentrated planar fiber supensions, J. Rheol. 49 (2005) 1029-1058.

[18] G. Ausias, X. Fan, R. Tanner, Direct simulation for concentrated fibre suspensions in transient and steady state shear flows, J. Non-Newtonian Fluid Mech. 135 (2006) 46-57. 
[19] P.J.J. Dumont, S. Le Corre, L. Orgéas, D. Favier, A numerical analysis of the evolution of bundle orientation in concentrated fibre-bundle suspensions, J. Non-Newtonian Fluid Mech. 160 (2009) 76-92

[20] S. Lomov, I. Verpoest, Model of shear of woven fabric and parametric description of shear resistance of glass woven reinforcements, Compos. Sci. Technol. 66 (2006) 919-933.

[21] M. Alkhagen, S. Toll, Micromechanics of a compressed fiber mass, J. Appl. Mech. 74 (2007) 723-731.

[22] E. Maire, J.-Y. Buffières, L. Salvo, J.-J. Blandin, W. Ludwig, J.-M. Létang, On the application of x-ray microtomography in the field of material science, Adv. Eng. Mater. 3 (2001) 539-546.

[23] S. Rolland du Roscoat, M. Decain, X. Thibaut, C. Geindreau, J.F. Bloch, Estimation of microstructural properties from synchrotron X-ray microtomography and determination of the $\mathrm{REV}$ in paper materials, Acta Mater. 55 (2007) 2841-2850.

[24] L. H. Switzer, D. J. Klingenberg, Rheology of sheared flexible fiber suspensions via fiber-level simulations, J. Rheol. 47 (3) (2003) 759-778.

[25] D. Durville, Numerical simulation of entangled materials mechanical properties, J. Mater. Sci. 40 (2005) 5941-5948.

[26] S. G. Advani, C. L. Tucker, The use of tensors to describe and predict fiber orientation in short fiber composites, J. Rheol. 3 (8) (1987) 751-784.

[27] Y. Leterrier, A. Pinyol, L. Rougier, J.H. Waller, J.-A.E. Månson, P.J.J. Dumont, J. Andersons, J. Modniks, M. Campo, P. Sauer, J. Schwenzel, Thin Solid Films 518 (2010) 6984-6992.

[28] S. Toll, Note: On the tube model for fiber suspensions, J. Rheol. 37 (1) (1993) $123-125$.

[29] J.P. Vassal, L. Orgéas, D. Favier, J.L. Auriault, S. Le Corre, Upscaling the diffusion equations in particulate media made of highly conductive particles. Part II: Application to fibrous materials, Phys. Rev. E 77 (2008) 011303. 
(a)
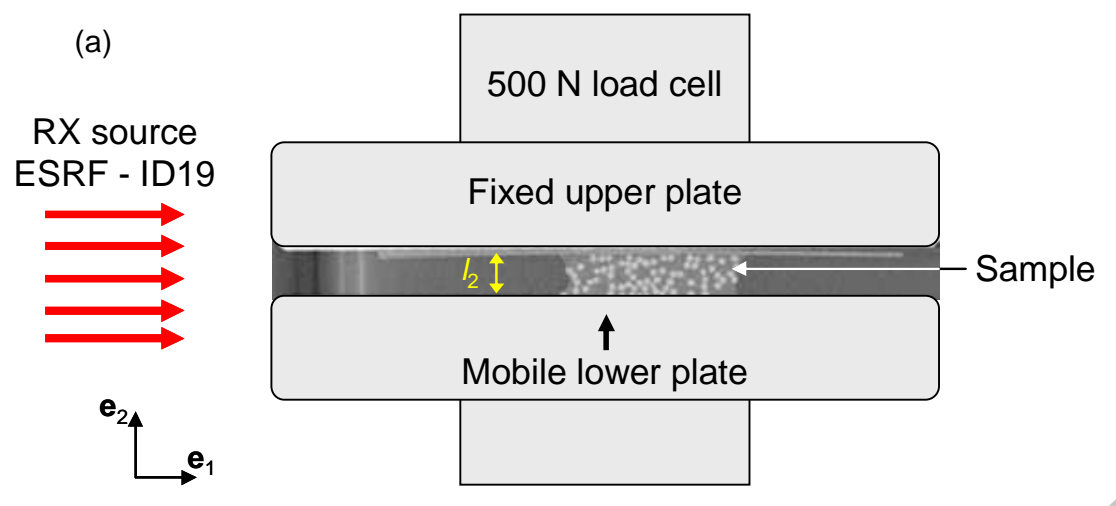

(b)

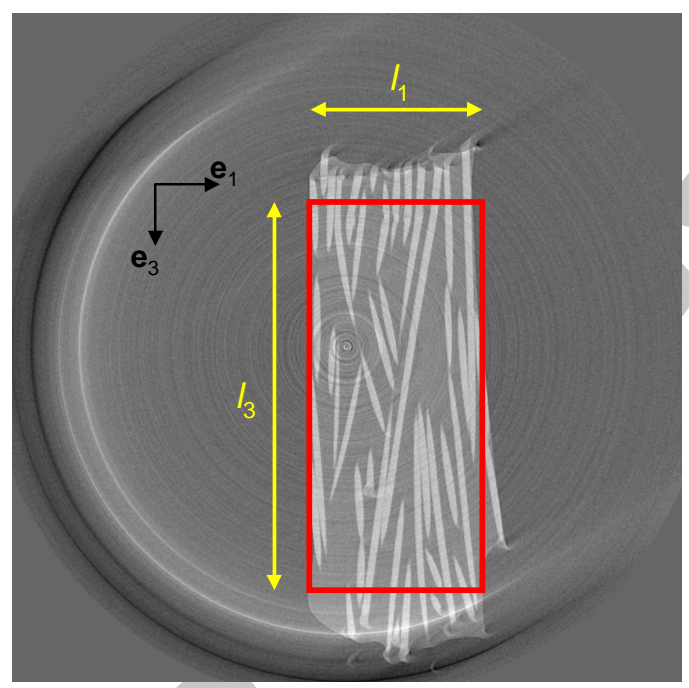

Fig. 1. Scheme of the micro-rheometer and the model fibre bundle (reconstructed slices) which were put inside the ESRF X-ray microtomograph: vertical cross section in a plane parallel to $\left(\mathbf{e}_{1}, \mathbf{e}_{2}\right)$ (a), horizontal cross section in a plane parallel to $\left(\mathbf{e}_{1}, \mathbf{e}_{3}\right)(\mathrm{b})$. 
Initial non-deformed configuration
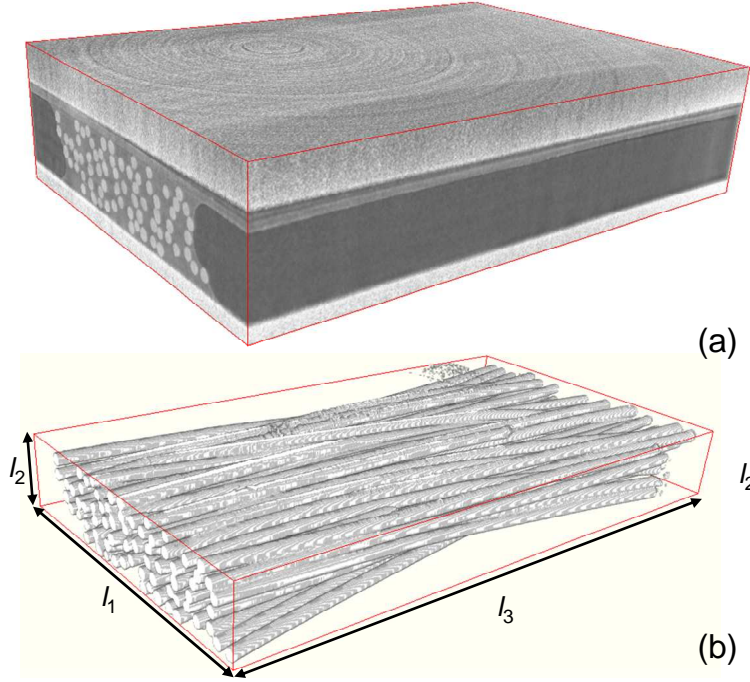

(b)
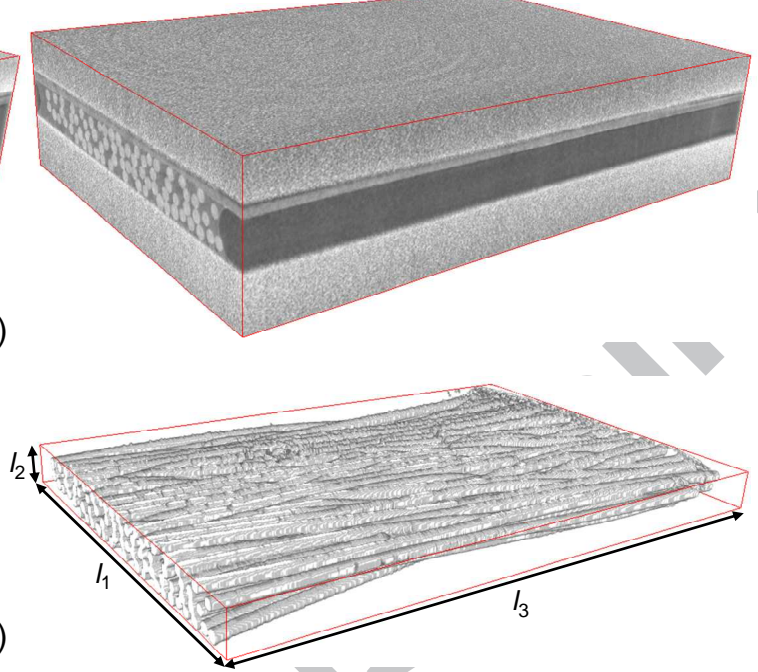

(c)

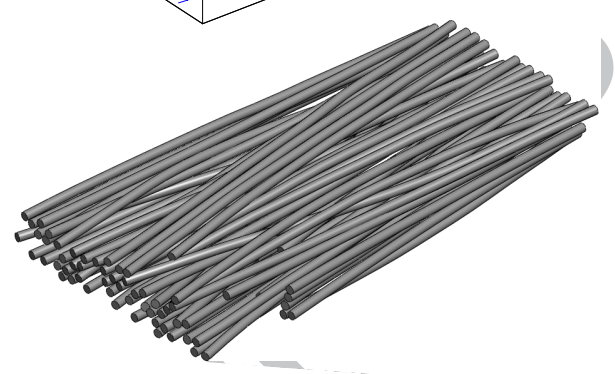

(d)
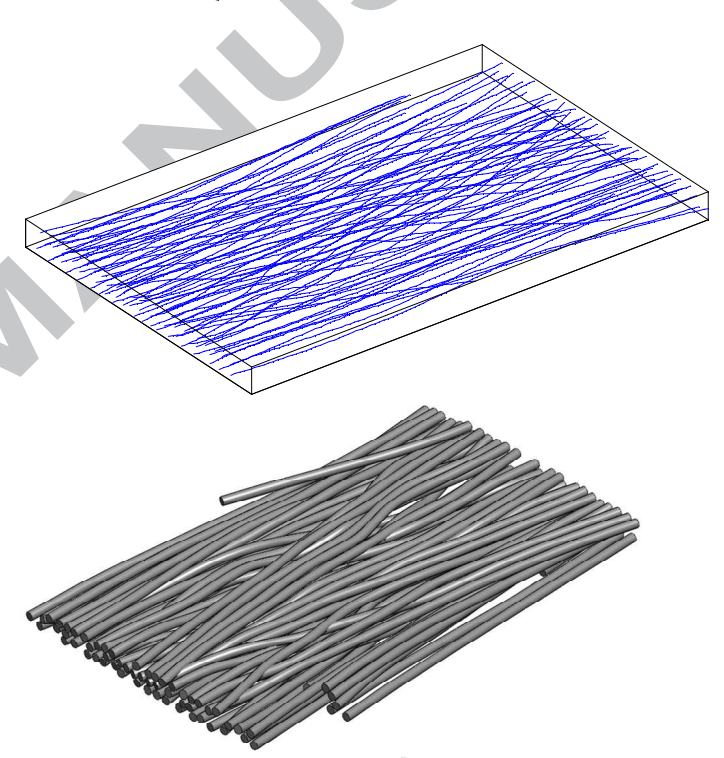

(e)

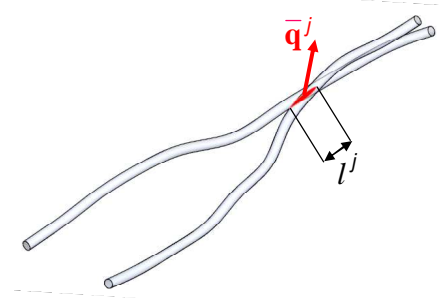

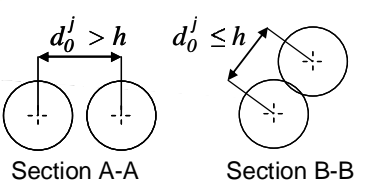

Fig. 2. Illustration of the various image analysis operations used to estimate microstructure descriptors. The left pictures concern the initial non-deformed state, the right ones the last step of compression: reconstructed 3D grey scale volumes (a), volumes of fibres $V$ after fibre segmentation (b), fibre centrelines (c), volumes of fibres $V$ after reconstruction (d), detection of a fibre-fibre contact between two contacting fibres (e). In the left picture (e), two sections (A-A and B-B) are sketched in order to illustrate distances $d_{0}^{j}$ and $h$ used to detect fibre-fibre contacts (here with $h=D$ ). 

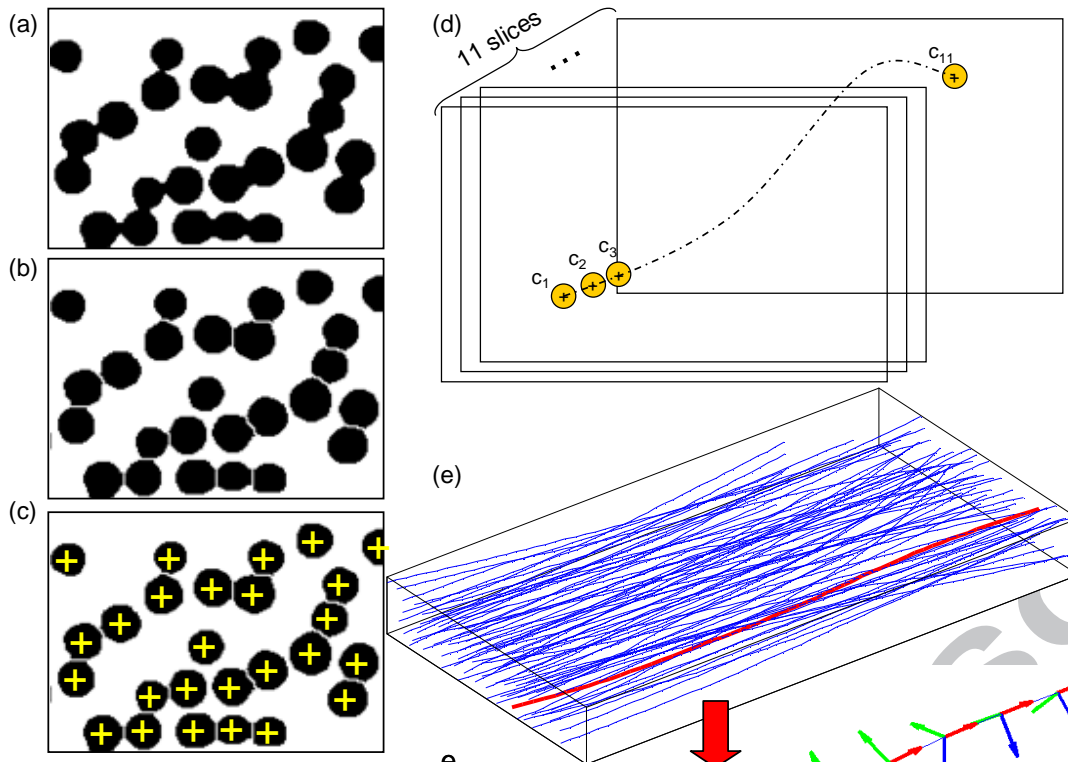

(f)

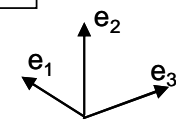

(e)
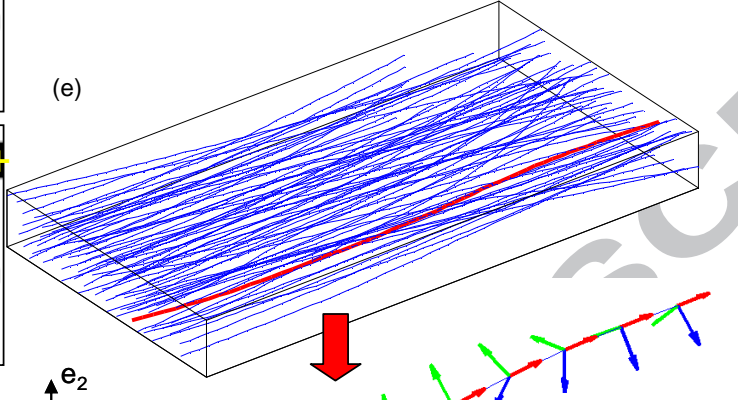

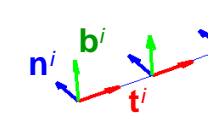

Fig. 3. Method used to detect and characterise the fibre centrelines in $V$ : (a) example of one of the 11 parallel slices shown in (d); (b) same slice after a 2D watershed; (c) same slice with the centres of mass of the cross sections; (d) generating the centreline of one fibre; (e) view of all centrelines; (f) local Frenet basis associated to one centreline.

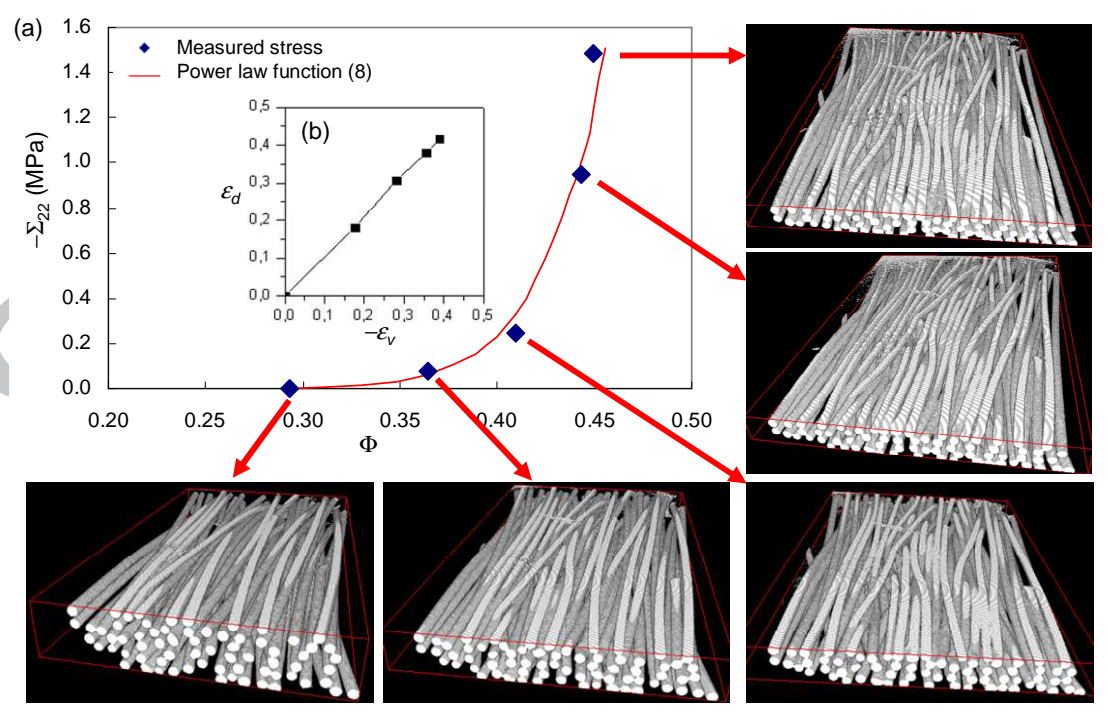

Fig. 4. Mechanical response at the mesoscale: evolution of the mean stress $\Sigma_{22}$ with the volume fraction of fibres $\Phi$ in $V$ (outer graph), evolution of the mean deviatoric strain $\varepsilon_{d}$ as a function of the mean volumetric strain $\varepsilon_{v}$. Around the graphs, 3D segmented volumes obtained at the five compression steps have been represented. 

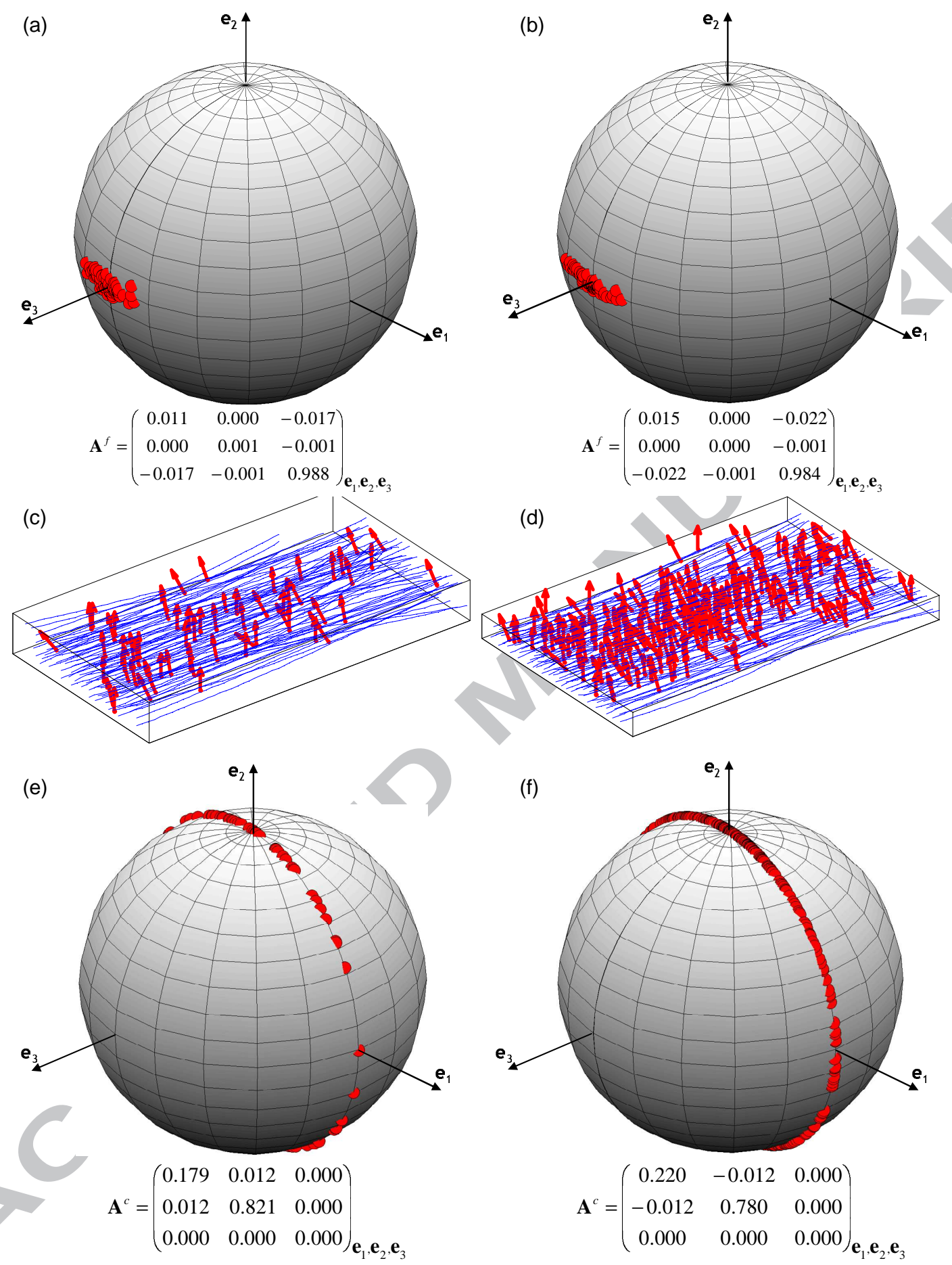

Fig. 5. Orientation fibres and fibre-fibre contacts. Orientation unit spheres and associated second order orientation tensors for the fibres (a-b) and for the fibre-fibre contacts (e-f). Pictures (c-d) give the fibre centrelines and the contact orientation vectors $\overline{\mathbf{q}}^{j}$ contained in $V$. The left pictures concern the initial non-deformed state, the right ones the last step of compression. 

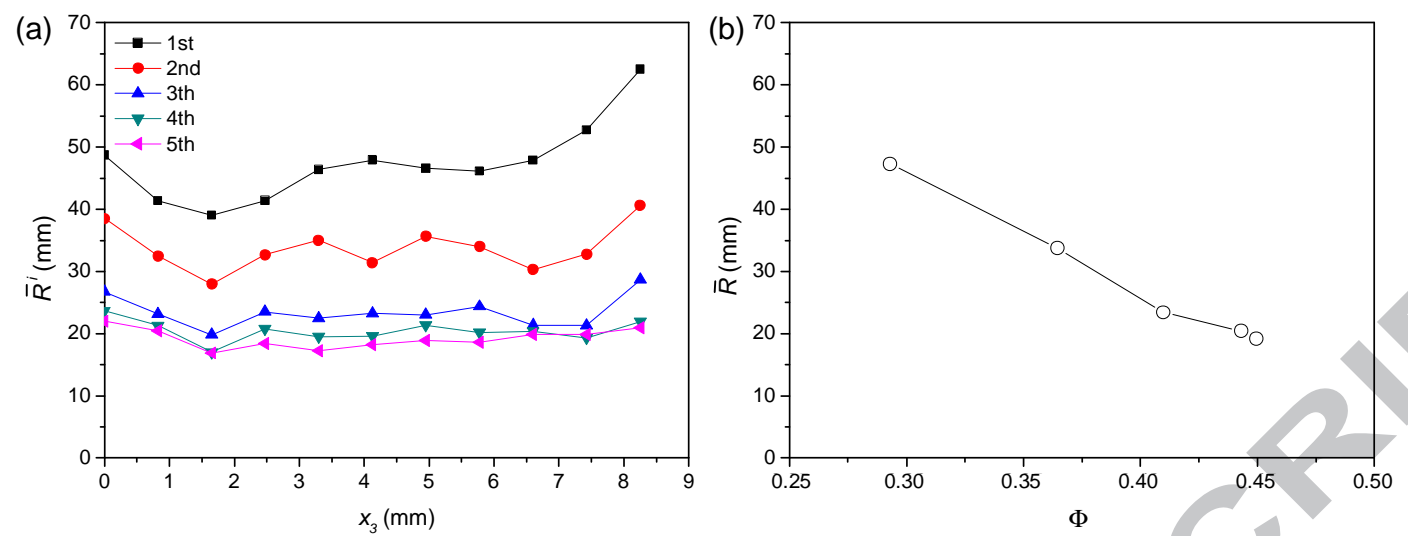

Fig. 6. Evolution (a) of the curvature radii $\bar{R}^{i}$ as a function of the position $x_{3}$ along the bundle for various compression states, (b) of the mean curvature radius $\bar{R}$ with the fibre content $\Phi$.

(a)

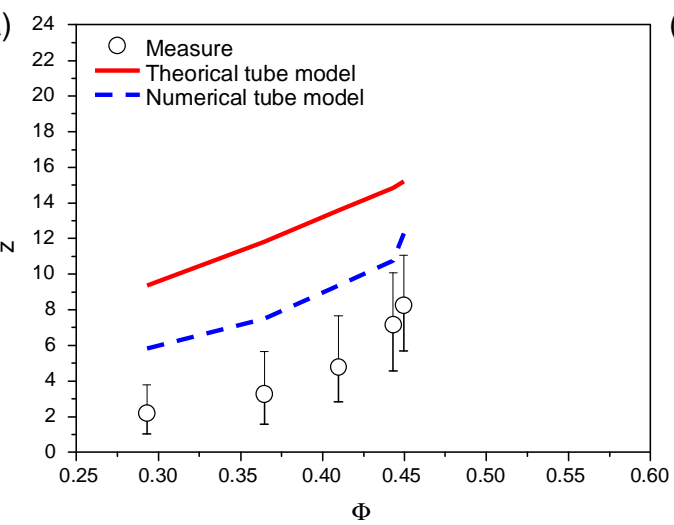

(2)

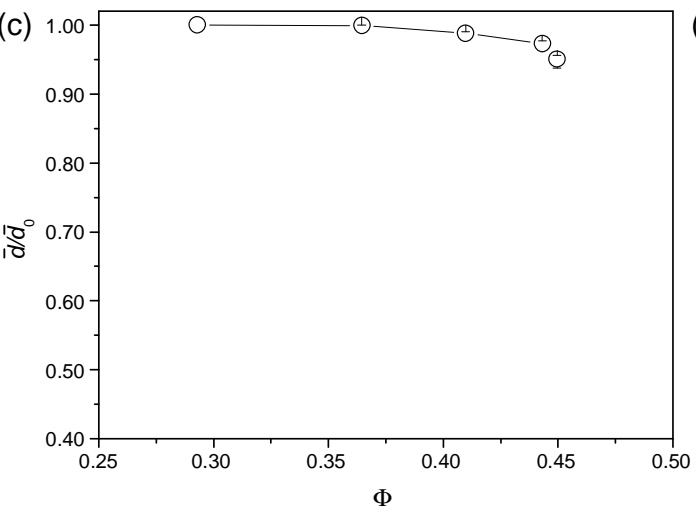

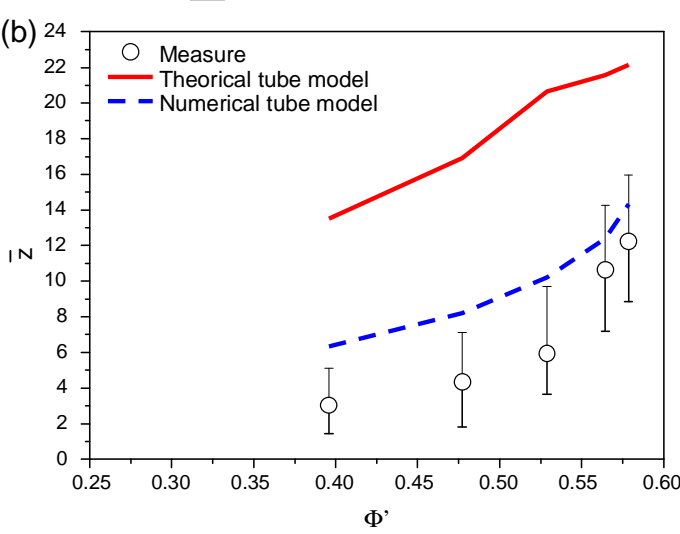

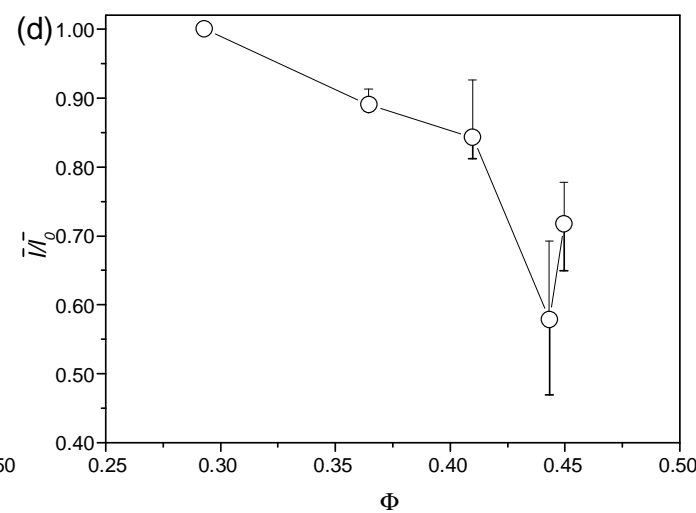

Fig. 7. Evolution of the average coordination numbers $\bar{z}$ (a) and $\bar{z}^{\prime}$ (b) with the fibre contents $\Phi$ (a) and $\Phi^{\prime}$ (b). Evolution of the dimensionless mean fibre-fibre distance $\bar{d} / \bar{d}_{0}\left(\bar{d}_{0} \approx 145 \pm 7.5 \mu \mathrm{m}\right)$ (c) and the dimensionless mean contact length $\bar{l} / \bar{l}_{0}\left(\bar{l}_{0} \approx 900 \pm 7.5 \mu \mathrm{m}\right)(\mathrm{d})$ with the fibre content $\Phi$. 